secretion by patient-derived monocytes, a mechanism with translational potential in SpA.

Conclusion: Our detailed characterization of Tregs at an important inflammatory site illustrates the marked specialization of Treg subpopulations and identifies a broad transcriptional profile upregulated across all synovial regulatory cells. Our TCR analysis provides evidence of Treg clonal expansion, which may be driven by antigen, and confirms functional specialisation of individual clones. We also propose a new insight into a Treg functional mechanism through LAG-3 that suggests a novel therapeutic approach to immune-driven diseases.

REFERENCES:

[1] Penkava et al., Nature Communications, 2020

Disclosure of Interests: Davide Simone: None declared, Frank Penkava: None declared, Anna Ridley: None declared, Stephen Sansom: None declared, Hussein Al Mossawi Employee of: UCB, Paul Bowness Grant/research support from: Regeneron, Celgene/BMS and GSK

DOI: 10.1136/annrheumdis-2021-eular.4278

\section{OP0033 $\quad$ REGULATORY T CELL CD39 EXPRESSION AS A PREDICTOR OF EARLY REMISSION-INDUCTION WITH METHOTREXATE IN NEW-ONSET RHEUMATOID ARTHRITIS}

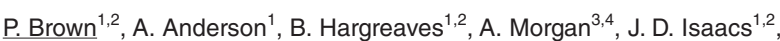
A. Pratt ${ }^{1,2} .{ }^{1}$ Translational and Clinical Research Institute, Faculty of Medical Sciences, Newcastle University, Newcastle upon Tyne, United Kingdom; ${ }^{2}$ Musculoskeletal Unit, Newcastle upon Tyne Hospitals NHS Foundation Trust, Newcastle upon Tyne, United Kingdom; ${ }^{3}$ Leeds Institute of Cardiovascular and Metabolic Medicine, Discovery and Translational Science Department, School of Medicine, University of Leeds, Leeds, United Kingdom; ${ }^{4}$ National Institute for Health Research, Leeds Biomedical Research Centre, Leeds, United Kingdom

Background: The long term outcomes for patients with rheumatoid arthritis (RA) depend on early and effective disease control. Methotrexate remains the key first line disease modifying therapy for the majority of patients, with $40 \%$ achieving an ACR50 on monotherapy(1). There are at present no effective biomarkers to predict treatment response, preventing effective personalisation of therapy. A putative mechanism of action of methotrexate, the potentiation of anti-inflammatory adenosine signalling, may inform biomarker discovery. By antagonism of the ATIC enzyme in the purine synthesis pathway, methotrexate has been proposed to increase the release of adenosine moieties from cells, which exert an anti-inflammatory effect through interaction with ADORA2 receptors(2). Lower expression of CD39 (a cell surface 5-'ectonucleotidase required for the first step in the conversion of ATP to adenosine) on circulating regulatory T-Lymphocytes (Tregs) was previously identified in patients already established on methotrexate who were not responding (DAS28 $>4.0$ vs <3.0)(3). We therefore hypothesised that pre-treatment CD39 expression on these cells may have clinical utility as a predictor of early methotrexate efficacy.

Objectives: To characterise CD39 expression in peripheral blood mononuclear cells in RA patients naïve to disease modifying therapy commencing methotrexate, and relate this expression to 4 variable DAS28CRP remission $(<2.6)$ at 6 months.

Methods: 68 treatment naïve early RA patients starting methotrexate were recruited from the Newcastle Early Arthritis Clinic and followed up for 6 months Serial blood samples were taken before and during methotrexate therapy with peripheral blood mononuclear cells isolated by density centrifugation. Expression of CD39 by major immune subsets (CD4+ and CD8+ T-cells, B-lymphocytes, natural killer cells and monocytes) was determined by flow cytometry. The statistica analysis used was binomial logistic regression with baseline DAS28CRP used as a covariate due to the significant association of baseline disease activity with treatment response.

Results: Higher pre-treatment CD39 expression was observed in circulating CD4+ T-cells of patients who subsequently achieved clinical remission at 6 months versus those who did not (median fluorescence 4854.0 vs 3324.2; $p=$ 0.0108 ; Figure 1-A). This CD39 expression pattern was primarily accounted for by the CD4+CD25 high sub-population (median fluorescence 9804.7 vs $6455.5 ; \mathrm{p}=$ 0.0065 ; Figure 1-B). These CD25 high cells were observed to have higher FoxP3 and lower CD127 expression than their CD39 negative counterparts, indicating a Treg phenotype. No significant associations were observed with any other circulating subset. A ROC curve demonstrates the discriminative utility of differential CD39 expression in the CD4+CD25 high population for the prediction of DAS28CRP remission in this cohort, showing greater specificity than sensitivity for remission prediction(AUC: 0.725; 95\% Cl: 0.53 - 0.92; Figure 1-C). Longitudinally, no significant induction or suppression of the CD39 marker was observed amongst patients who did or did not achieve remission over the 6 months follow-up period.

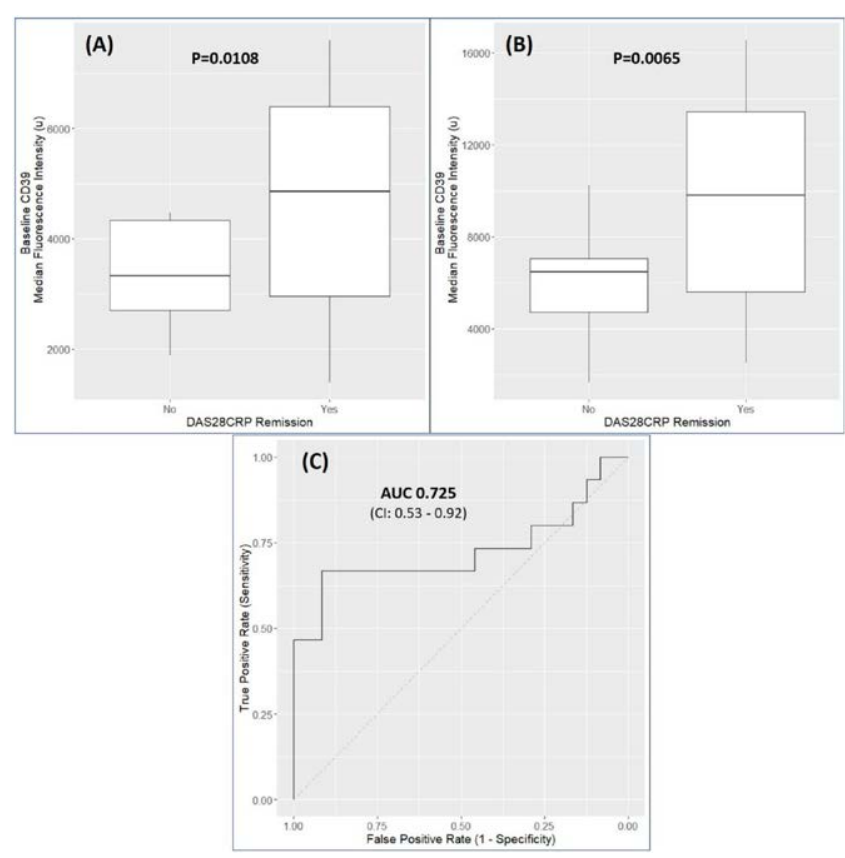

Figure 1. Six month DAS28CRP remission versus pre-treatment median fluorescence of CD39 expression on CD4+ T-cells (A); CD25 High expressing CD4+ T-cells (B); and ROC curve of predictive utility of pre-treatment CD39 expression on CD25 High CD4+ T-cells (C).

Conclusion: These findings support the potential role of CD39 in the mechanism of methotrexate response. Expression of CD39 on circulating Tregs in treat ment-naïve RA patients may have particular value in identifying early RA patients likely to respond to methotrexate, and hence add value to evolving multi-parameter discriminatory algorithms.

\section{REFERENCES:}

[1] Hazlewood GS, et al. BMJ. 2016 21;353:11777

[2] Brown PM, et al. Nat Rev Rheumatol. 2016;12(12):731-742

[3] Peres RS, et al. Proc Natl Acad Sci U S A. 2015;112(8):2509-2514

Disclosure of Interests: None declared

DOI: 10.1136/annrheumdis-2021-eular.2030

\section{OP0034 STP938, A NOVEL, POTENT AND SELECTIVE INHIBITOR OF CTP SYNTHASE 1 (CTPS1) DEMONSTRATES EFFICACY IN RODENT MODELS OF INFLAMMATION AND ARTHRITIS}

H. Asnagli ${ }^{1}$, A. Novak ${ }^{2}$, L. Birch ${ }^{2}$, R. Lane ${ }^{2}$, N. Minet ${ }^{3}$, D. Laughton ${ }^{2}$, P. George ${ }^{4}$ G. De Ribains ${ }^{5}$, S. Latour ${ }^{3}$, A. Fischer ${ }^{6}$, T. Bourne ${ }^{7}$, A. Parker ${ }^{1}$. ${ }^{1}$ Step Pharma, Pharmacology, Archamps, France; ${ }^{2}$ Sygnature Discovery, Discovery BuildingBiocity Building, Nottingham, United Kingdom; ${ }^{3}$ Institut Imagine, Laboratory of Lymphocyte Activation \& EBV Susceptibility, INSERM-U1163, Paris, France; ${ }^{4}$ Independent consultant, Drug Discovery \& Development, Paris, France; ${ }^{5}$ Gamut, Therapeutics, Paris, France; ${ }^{6}$ INSERM-U1163, AP-HP, Paris, France; ${ }^{7}$ Milvuswood, Consultancy, High Wycombe, United Kingdom

Background: The final rate-limiting step in pyrimidine synthesis is the conversion of UTP to CTP which is catalyzed by cytidine triphosphate synthase 1 (CTPS1) or CTPS2. A hypomorphic mutation in the CTPS1 gene has highlighted the essential and non-redundant role of CTPS1 in T and B lymphocyte proliferation ${ }^{1}$. These patients exhibit no effects on non-hematopoietic tissues. Thus, selective inhibition of CTPS1 represents a novel targeted approach to dampen pathological T- and B-cell lympho-proliferation. STP938 is an orally bioavailable, small molecular weight, selective inhibitor of CTPS1 developed by Step Pharma Objectives: To demonstrate the in vitro effects of CTPS1 inhibition on T and B cell proliferation and the therapeutic potential of STP938 using in vivo models of disease.

Methods: The in vitro anti-proliferative activity of STP938 was investigated using cell lines and primary human PBMCs. STP938 was assessed in vivo using the $\mathrm{DTH}-\mathrm{KLH}$ rat model and the mouse collagen-induced arthritis (CIA) model. For the KLH-DTH model, Lewis rats were immunized with $\mathrm{KLH}$, a week later, challenged locally at the ear with KLH antigen, ear swelling was assessed after 24 hours. Blood samples were collected for detection of KLH-specific IgG levels at day 8. STP938 was given orally one-hour prior to immunization and then b.i.d. for 7 days. For the CIA model, DBA-1 mice were immunized with Collagen type II and complete Freund's adjuvant and received a booster immunization three 
weeks later. STP938 was administered to mice developing signs of arthritis from Day 28 to 45 orally daily b.i.d.

Results: STP938 inhibited in vitro proliferation of $\mathrm{HEK}_{\mathrm{wt}}$ but not HEK-CTPS $1_{\mathrm{KO}}$ cells as well as Jurkat and human PBMCs. STP938 demonstrated a significant and dose-dependent inhibition of KLH-specific T and B cell responses in vivo. STP938 significantly reduced the disease severity in the CIA model in a dose-dependent manner as determined by clinical and histopathological readouts. Conclusion: Our preliminary in vitro and in vivo results indicate that inhibition of CTPS1 specifically blocks proliferation of cells derived from the lymphocyte lineage and reduces the $T$ cell driven inflammatory response. These data highlight the therapeutical potential of STP938 in treating patients with autoimmune diseases such as rheumatoid arthritis.

\section{REFERENCES:}

[1] Martin et al, JCl Insight. 2020, 12;5(5):133880

Disclosure of Interests: Hélène ASNAGLI Employee of: Step Pharma, Andrew Novak: None declared, Louise Birch Shareholder of: Step Pharma, Rebecca Lane: None declared, Norbert Minet Employee of: employee as Ph D student under CIFRE grant, David Laughton: None declared, Pascal George Shareholder of: Step Pharma, Geoffroy de Ribains Shareholder of: as former employee of Step Pharma, Employee of: former employee of Step Pharma, Sylvain Latour: None declared, Alain Fischer: None declared, Tim Bourne Shareholder of: UCB, Step Pharma, Sitryx Therapeutics, Consultant of: a range of biotech companies, Employee of: former employee of Step Pharma and Sitryx Therapeutics, Andrew Parker Employee of: Step Pharma

DOI: 10.1136/annrheumdis-2021-eular.148

\section{OP0035 IN PATIENTS WITH RHEUMATOID ARTHRITIS USING COLONIC TISSUES AND SERA}

R. Audo ${ }^{1,2}$, P. Sanchez ${ }^{1}$, J. Mielle ${ }^{2}$, L. Macia ${ }^{3}$, B. Rivière ${ }^{4}$, C. Lukas ${ }^{1}$, B. Combe ${ }^{1}, \mathrm{~J}_{\text {. Morel }}{ }^{1}, \mathrm{C}$. Daien ${ }^{1}$. ${ }^{1} \mathrm{CHU}$ Montpellier, Department of Rheumatology, Montpellier, France; ${ }^{2}$ UMR5535, IGMM, Montpellier, France; ${ }^{3}$ University of Sydney, Charles Perkins Center, Montpellier, France; ${ }^{4} \mathrm{CHU}$ Montpellier, Laboratoire d'Anatomie et de Cytologie Pathologiques, Montpellier, France

Background: Patients with rheumatoid arthritis (RA) have an altered gut microbiota (dysbiosis) (1-3). This microbiota interacts with intestinal epithelium which can lead to an increased intestinal permeability, responsible for the passage of antigens and inflammatory molecules, and can therefore promote systemic inflammation. Gut microbiota tends to normalize with disease control (2), suggesting that systemic inflammation may directly influence the composition of microbiota and the gut barrier. It was shown in many inflammatory diseases that intestinal permeability is impaired, but to date there is very little data in RA.

Objectives: In the present study, we evaluate the intestinal permeability in RA patients by analyzing tight junctions in colonic biopsies and serum markers.

Methods: Colonic biopsies from 20 RA patients who underwent coloscopy for screening with normal histology were compared with those from 20 age and sex matched controls. ZO-1, occludin and claudin 2 junction proteins were evaluated by immunohistochemistry. The staining intensity was assessed by two blinded independent readers. The serum concentrations of LPS-binding protein (LBP), CD14s and zonulin were evaluated by ELISA in 25 patients naive of DMARDs, 41 patients before and after introduction of a DMARDs and 21 controls. Elevated zonulin in serum indicates an increase in intestinal permeability while LBP and CD14s indicate bacterial translocation.

Results: ZO-1 expression was significantly lower in biopsies from patients with RA than controls (mean score $\pm S D$ of $1.6 \pm 0.56$ vs $2.0 \pm 0.43 ; p=0.01$ ). Age, sex, disease duration and immunological status did not significantly influence the expression of colonic junction proteins. LBP and CD14s were higher in serum from RA patients naive of DMARDs than controls $(p=0.002$ and $p=0.003)$. LBP, CD14s and zonulin levels significantly correlated with DAS28 ( $r=0.61, p=0.005 ; r=0.51, p=0.030$ and $r=0.46, p=0.049$, respectively). After treatment, unlike non-responders, LBP and CD14s were significantly reduced in DMARD responders and variations in LBP and CD14s significantly correlated with changes in DAS28 $(r=0.46, p=0.002$ and $r=0,33$ and $\mathrm{p}=0.030$, respectively).

Conclusion: This work is one of the first to explore intestinal permeability in RA and to show altered tight junction in colonic tissue from RA. This increased intestinal permeability appears to be related to the systemic inflammation. Improving the gut microbiota through food or probiotics could enhance the effect of treatments by limiting this amplification loop of inflammation. REFERENCES:

[1] Horta-Baas G, Romero-Figueroa MDS, Montiel-Jarquin AJ, Pizano-Zarate ML, Garcia-Mena J, Ramirez-Duran N. Intestinal Dysbiosis and Rheumatoid Arthritis: A Link between Gut Microbiota and the Pathogenesis of Rheumatoid Arthritis. J Immunol Res. 2017;2017:4835189.
[2] Zhang X, Zhang D, Jia H, Feng Q, Wang D, Liang D, et al. The oral and gut microbiomes are perturbed in rheumatoid arthritis and partly normalized after treatment. Nat Med. 2015;21(8):895-905

[3] Maeda Y, Kurakawa T, Umemoto E, Motooka D, Ito Y, Gotoh K, et al. Dysbiosis Contributes to Arthritis Development via Activation of Autoreactive T Cells in the Intestine. Arthritis Rheumatol. 2016;68(11):2646-61.

Disclosure of Interests: Rachel Audo: None declared, Pauline Sanchez: None declared, Julie Mielle: None declared, Laurence Macia: None declared, Benjamin Rivière: None declared, Cédric Lukas: None declared, Bernard Combe: None declared, Jacques Morel: None declared, Claire Daien Speakers bureau: Pfizer roche chugai fresenius BMS msd Novartis galapagos, Consultant of: Abivax abbbvie BMS roche chugai, Grant/research support from: Pfizer, roche-chugai, fresenius, msd

DOI: 10.1136/annrheumdis-2021-eular.2642

\begin{tabular}{|l|l|}
\hline OP0036 & IL-6 ACTIVATES YES-ASSOCIATED PROTEIN \\
& (YAP) IN FIBROBLASTS AND INDUCES YAP-SNAIL \\
& COMPLEX FORMATION TO DRIVE SYNOVIAL LINING \\
& PATHOLOGY IN INFLAMMATORY ARTHRITIS
\end{tabular}

R. Symons ${ }^{1}$, F. Colella ${ }^{1}$, F. Collins ${ }^{1}$, A. Roelofs ${ }^{1}$, C. De Bari ${ }^{1}{ }^{1}$ Arthritis and Regenerative Medicine Laboratory, Institute of Medical Sciences, University of Aberdeen, Aberdeen, United Kingdom

Background: In rheumatoid arthritis (RA), the fibroblast-like synoviocytes (FLS) in synovial lining become invasive and cause joint destruction. The molecular mechanisms underpinning this pathogenic FLS phenotype are incompletely understood. The FLS descend from Growth differentiation factor 5 (Gdf5)-expressing joint interzone cells in the embryo, and we showed that conditional ablation of the transcriptional co-activator Yes associated protein (Yap) in Gdf5-lineage cells prevents synovial lining hyperplasia after traumatic cartilage injury in mice [1].

Objectives: Here, we investigated a potential role for Yap in pathogenic FLS in immune-mediated inflammatory arthritis.

Methods: Immunohistochemistry was used to detect Yap in human RA synovium and Yap, Snail and Ctgf in mouse synovium following antigen-induced arthritis (AIA). To determine the effect of Yap knockout (KO) in synovial stroma cells, AIA was induced in Gdf5-Cre;tdTomato;Yap ${ }^{\text {fl/fl }}$ (Yap cKO) and Gdf5-Cre;tdTomato;Yap ${ }^{\text {wt/wt }}$ (control) mice, or in Pdgfra-CreER;Yap ${ }^{\text {fl/fl }}$ (Yap ciKO, targeting Pdgfro-expressing fibroblasts) and Yap ${ }^{\text {fl/fl }}$ or Yap ${ }^{\text {WT/fl }}$ (control) mice after adult tamoxifen induction. Yap KO in both models was confirmed by immunohistochemistry. After nine days, arthritis severity was determined by histological scoring of synovial lining hyperplasia, immune infiltrates, cellular exudate, and marginal erosions. TdTomato+ Gdf5-lineage cells in synovium were quantified. In vitro, Yap reporter cells were treated with inflammatory cytokines to evaluate their ability to stimulate Yap-induced GFP expression by flow cytometry. Snail overexpression, siRNA-mediated Yap knockdown, and IL-6/sIL-6R stimulation were performed on normal mouse FLS, AIA-FLS or human RA-FLS, and cell invasion through a matrigel-coated transwell was quantified. A proximity ligation assay was utilised to detect Yap/Snail complex formation.

Results: Average expression levels of Yap $(p<0.0001)$, its transcription factor partner Snail $(p=0.002)$, and their downstream target Ctgf $(p=0.0003)$, were increased in mouse synovium after AIA $(n=5)$, and Yap was highly expressed by FLS in human RA synovium. Yap cKO mice $(n=24)$ showed a significantly decreased arthritis severity $(p=0.002)$ after AIA compared to controls $(n=22)$, with significant reductions in synovial lining hyperplasia $(p<0.001)$, synovial immune cell infiltrates $(p=0.026)$ and marginal erosions $(p=0.002)$. Similarly, Yap ciKO mice $(n=6)$ showed a significant decrease in arthritis score $(p=0.039)$ after AIA compared to controls $(n=9)$. However, both control mice $(p<0.001)$ and Yap cKO mice $(p<0.001)$ showed an extensive expansion of tdTomato+ Gdf5-lineage synovial cells after AIA, with no significant difference between control and Yap cKO mice. In vitro, Yap knockdown prevented IL-6/sIL-6R-induced invasion of normal mouse FLS $(p=0.037)$ and decreased the invasiveness of AIA-FLS $(p=0.0057)$. Using Yap reporter cells, we found that Yap was activated by IL-6/sIL-6R ( $p=0.016)$, but not TNF $\alpha$ or IL-1 $\beta$. Finally, IL-6/sIL-6R treatment of normal mouse FLS $(p=0.033)$ or human RA-FLS $(p=0.036)$ induced Yap-Snail complex formation, and Yap knockdown prevented FLS invasion induced by Snail overexpression $(p=0.027)$.

Conclusion: These data demonstrate that via activation by IL-6, and co-operation with the transcription factor Snail, Yap acts as a key modulator of the invasive and destructive phenotype of FLS in inflammatory arthritis. Therapeutic targeting of Yap could reduce joint destruction in RA.

\section{REFERENCES:}

[1] A. J. Roelofs et al., "Joint morphogenetic cells in the adult mammalian synovium,” Nat. Commun., vol. 8, no. May, p. 15040, 2017. DOI: 10.1136/ annrheumdis-2018-213799

Acknowledgements: This work was funded by the Medical Research Council (MR/L020211/1 and MR/L022893/1) and Versus Arthritis (20775 and 21156).

Disclosure of Interests: None declared

DOI: 10.1136/annrheumdis-2021-eular.2530 